\title{
Low temperature Mössbauer studies on magnetic nanocomposites
}

\author{
K A MALINI, M R ANANTHARAMAN* and AJAY GUPTA ${ }^{\dagger}$ \\ Department of Physics, Cochin University of Science and Technology, Cochin 682 022, India \\ ${ }^{\dagger}$ IUC-DAEF, Indore 452 017, India
}

MS received 2 January 2004; revised 14 June 2004

\begin{abstract}
Nanocomposites with magnetic components possessing nanometric dimensions, lying in the range 1-10 nm, are found to be exhibiting superior physical properties with respect to their coarser sized counterparts. Magnetic nanocomposites based on gamma iron oxide embedded in a polymer matrix have been prepared and characterized. The behaviour of these samples at low temperatures have been studied using Mössbauer spectroscopy. Mössbauer studies indicate that the composites consist of very fine particles of $\gamma-\mathrm{Fe}_{2} \mathrm{O}_{3}$ of which some amount exists in the superparamagnetic phase. The cycling of the preparative conditions were found to increase the amount of $\gamma-\mathrm{Fe}_{2} \mathrm{O}_{3}$ in the matrix.
\end{abstract}

Keywords. Magnetic nanocomposites; Mössbauer spectroscopy; ion exchange; $\gamma$-Fe $\mathrm{Fe}_{2} \mathrm{O}_{3}$; superparamagnetism.

\section{Introduction}

Magnetic nanocomposites may find potential applications in the recording industry for achieving high density information storage and in the refrigeration industry (Dagani 1992; Ziolo et al 1992). They are also used in colour imaging, ferrofluids, catalysts etc. Magnetic nanocomposites consisting of iron oxide embedded in polymer matrixes are found to be behaving like transparent magnets with remarkable electrical and optical properties (Ziolo et al 1992). The particle size effects dominate the magnetic properties of magnetic nanocomposites and the effect becomes more prominent when the particle size decreases. Nanophase materials and nanocomposites, characterized by an ultra fine grain size $(<50 \mathrm{~nm})$ have created a great deal of interest in recent years by virtue of their unusual mechanical, electrical, optical and magnetic properties (Chakravorty 1992; Ziolo et al 1992). The magnetic nanocomposites in which the magnetic nanoparticles are dispersed in nonmagnetic matrices have been found to possess entirely different magnetic characteristics with respect to their bulk counterparts (Chakravorty 1992; Dagani 1992; Shull and Bennet 1992; Ziolo et al 1992). The reduction in size modifies the magnetic order in these materials. The magnetic nanocomposites can be prepared by isolating the magnetic regions in nonmagnetic matrixes by precipitating from some solid solutions. The gross magnetic behaviour of a magnetic nanocomposite will be either paramagnetic or superparamagnetic. Because of the ease in controlling the magnetic behaviour by adjusting the processing parameters, such materials present a greater possibility for designing materials with specific properties.

\footnotetext{
*Author for correspondence
}

Magnetic nanocomposites containing $\gamma-\mathrm{Fe}_{2} \mathrm{O}_{3}$ have been prepared by the ion exchange method and the effect of cycling of preparation procedure on the magnetic properties of these nanocomposites have been studied. The results have already been reported elsewhere (Malini et al 2001). The low temperature behaviour has been investigated with a view to understanding the superparamagnetic/single domain characteristics of the magnetic component on the matrix.

According to Bean and Livingstone (1959) there are three kinds of magnetic structures for small particles: multidomain, single domain and quasidomain. A superparamagnetic particle is a single domain particle whose direction of magnetization fluctuates by thermal activation and attains thermal equilibrium with the applied field. Superparamagnetism is analogous to paramagnetism with the main difference being that the magnetic moment involved is not that of a single atom but of a single domain which may contain $10^{5}$ or more atoms ferromagnetically coupled by exchange forces. Thus in superparamagnetism the behaviour of single domain particles in thermodynamic equilibrium is identical to atomic paramagnetism except that the moments involved are very large (Bean 1955; Bean and Livingstone 1959). Thermal fluctuations will cause the direction of magnetization to undergo random reorientation. For non-interacting particles the magnetic susceptibility of the assembly of particles is the same as that of a classical Langevin paramagnetism with the magnetic moment of each particle equal to that of a large number of atoms. If the particles are fixed in space thermal activation is the mechanism for achieving thermodynamic equilibrium. The relaxation time, $\tau$, required for the assembly to achieve equilibrium increases exponentially with $K V / k T$. This is of the form

$$
\tau=\tau_{0} \exp (K V / k T)
$$


where $K$ is the anisotropy constant, $T$ the temperature and $k$ the Boltzmann constant (Morup 1994). In an assembly of particles the relaxation time of particles with smaller volume is short and their response is fast under the influence of an external magnetic field. Larger particles are magnetically frozen.

For particles of volume, $V$, there is a critical temperature called blocking temperature $\left(T_{\mathrm{B}}\right)$ below which the magnetic moments are fixed, i.e. their approach to thermodynamic equilibrium is blocked.

If the time of the experiment is around $100 \mathrm{~s}$, then the blocking temperature is $T_{\mathrm{B}}=K V / 25 k_{\mathrm{B}}$ and the critical volume is $V_{\mathrm{c}}=25 k_{\mathrm{B}} T / K$. Only particles with volume less than $V_{\mathrm{c}}$ will respond superparamagnetically to an external field at temperature, $T$.

Most of the reported literature indicates that magnetic nanocomposites do not show hysteresis at room temperature and hysteresis is observed only below the blocking temperature which is normally well below room temperature (Shull et al 1990, 1991; Vassiliou et al 1993; Ziolo et al 1993; Hsu and Huang 1995). But there also exist reports on nanocomposites with blocking temperature much above room temperature. Ambrose et al (1992) reported $T_{\mathrm{B}}$ to be much above room temperature in $\mathrm{Fe}-\mathrm{Al}_{2} \mathrm{O}_{3}$ systems. Roy et al (1993) have also reported $T_{\mathrm{B}}$ to be above room temperature in $\mathrm{Fe} / \mathrm{SiO}_{2}$ system. However, Shull, Ziolo and others have reported $T_{\mathrm{B}}$ to be well below room temperature (Shull et al 1990, 1991; Vassiliou et al 1993; Ziolo et al 1993; Hsu and Huang 1995).

${ }^{57} \mathrm{Fe}$ Mössbauer spectra of the sample were recorded at room temperature using a constant acceleration Mössbauer spectrometer and the spectrum was fitted to obtain the quadrupole splitting and isomer shift. The spectra were recorded at RT, $150 \mathrm{~K}$ and $100 \mathrm{~K}$.

Low temperature magnetic measurements on one representative sample was carried out and it was observed that the magnetization increases with decrease in temperature. It was found to be in accordance with the normal theoretical approach. That is, as the temperature decreases more and more ordering of moments come in and hence magnetization increases.

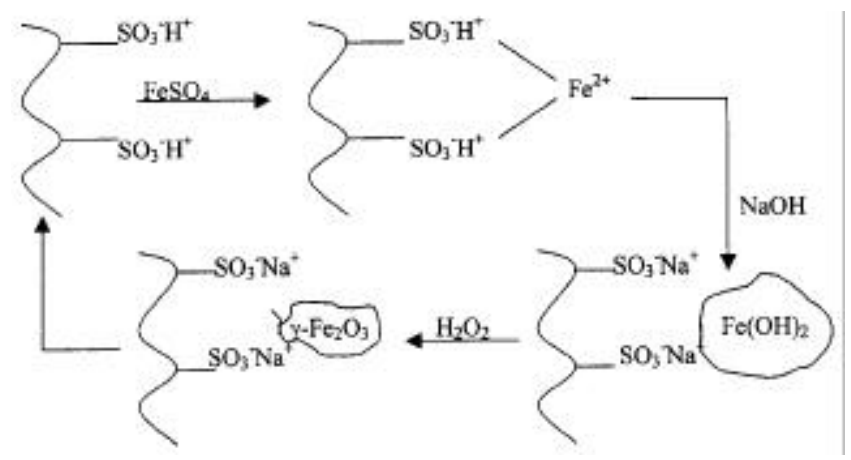

Figure 1. Scheme of preparation of nanocomposites.

\section{Experimental}

\subsection{Sample preparation}

Nanometer sized $\gamma-\mathrm{Fe}_{2} \mathrm{O}_{3}$ particles were embedded in a polymer matrix by the method of ion exchange followed by reduction. The method adopted here is a modification of the preparation scheme reported by Ziolo et al (1992) and Malini et al (2001). The polymer matrix employed is a cross linked polymer of sulphonated polystyrene and divinyl benzene, which have exchangeable $\mathrm{H}^{+}$ion containing $\mathrm{SO}_{3}^{-} \mathrm{H}^{+}$groups.

The ion exchange resin was exchanged with Fe ions from an aqueous solution of ferrous sulphate and these exchanged $\mathrm{Fe}$ ions were then converted into $\mathrm{Fe}(\mathrm{OH})_{2}$. This was then oxidized to yield, presumably, $\gamma-\mathrm{Fe}_{2} \mathrm{O}_{3}$. Drop wise addition of dilute aqueous solution of hydrogen peroxide $\left(\mathrm{H}_{2} \mathrm{O}_{2}\right)$ accelerated the conversion to oxide. The resin was then washed with water and then dried. Instead of adding $\mathrm{H}_{2} \mathrm{O}_{2}$, the addition of hydrazine hydrate solution was also tried for the conversion of hydroxide into oxide. The preparation scheme can be depicted as shown in figure 1.

Cycling of the preparation procedures was also done on one sample of strong ion exchange resin. The samples after first, eighth and twelfth cycles were taken. These cycled samples are labelled as SA1, SA8 and SA12 for 1 st, 8th and 12th cycled samples, respectively. It is found that by cycling the preparation steps more and more sites are exchanged by $\mathrm{Fe}$ ions and hence the possibility of incorporating more $\mathrm{Fe}_{2} \mathrm{O}_{3}$ in the matrix. The details are reported elsewhere (Malini et al 2001). The sample dealt with in this paper is a 12 cycled sample named SA12.

\subsection{Low temperature characterization}

The prepared sample SA12 was magnetically characterized by using VSM (model PARC 4200). The low temperature magnetization values as well as the thermomagnetization curve were obtained for the SA12 sample. The ${ }^{57} \mathrm{Fe}$

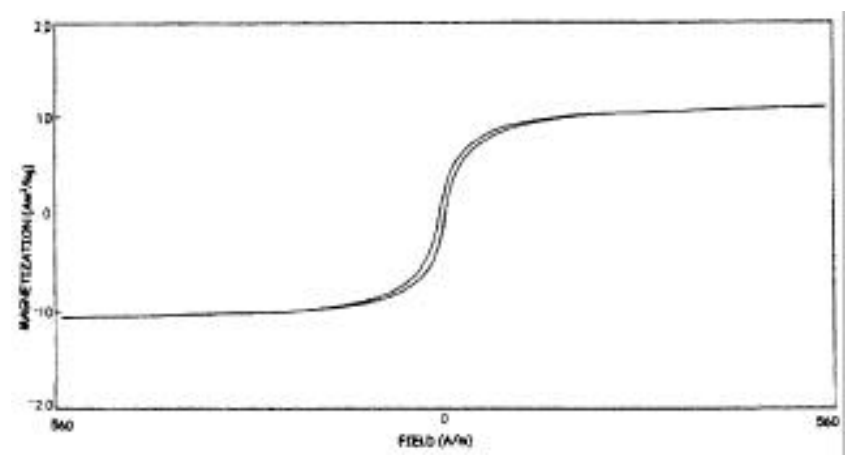

Figure 2. Room temperature hysteresis loop of the sample. 
Mössbauer spectra of the sample were recorded at room temperature and low temperature by using a constant acceleration Mössbauer spectrometer and the spectrum was fitted to obtain the quadrupole splitting and isomer shift.

\section{Results and discussion}

\subsection{Low temperature magnetization studies}

The prepared sample was magnetically characterized and the room temperature hysteresis loop of the sample is shown in figure 2 . The temperature dependence of saturation magnetization of the SA12 sample was studied. The saturation magnetization values are plotted against $T^{3 / 2}$ and figure 3 shows the temperature dependence of saturation magnetization. The curve clearly indicates that the system obeys Bloch law given by

$$
\sigma_{\mathrm{s}}=\sigma_{0}\left(1-B T^{3 / 2}\right)
$$

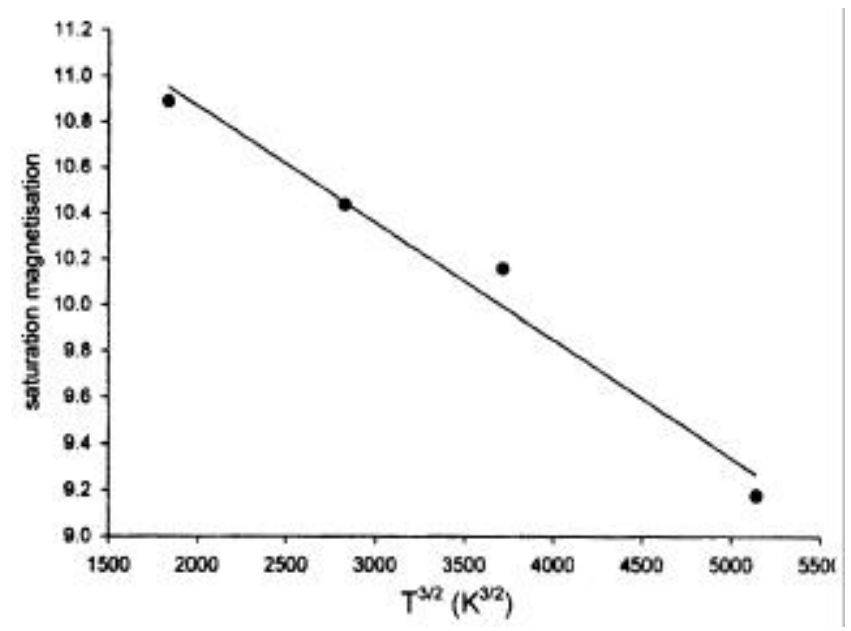

Figure 3. $T^{3 / 2}$ dependence of saturation magnetization.

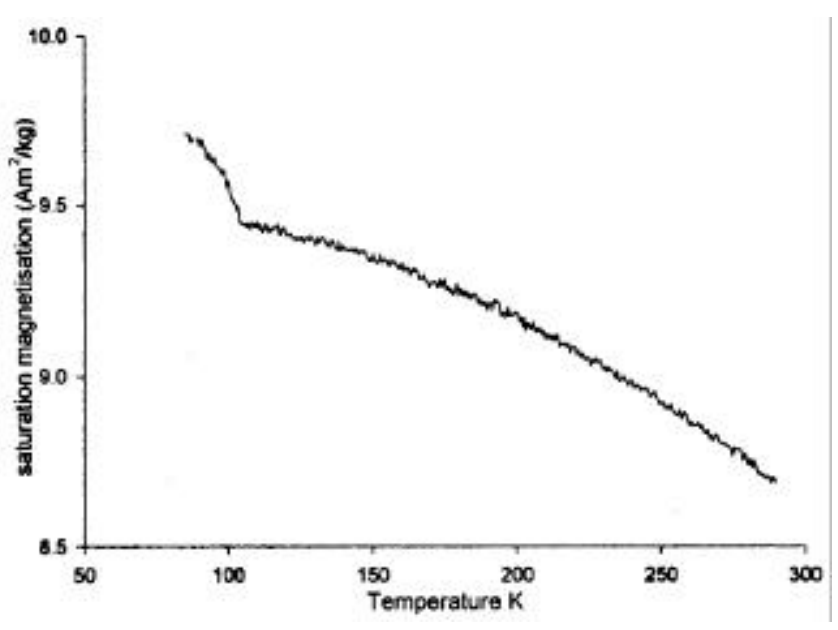

Figure 4. Thermomagnetization curve for SA12. where $B$ is called the Bloch constant or spin wave constant. The present system is found to be obeying the Bloch law above $120 \mathrm{~K}$ and there is a deviation from Bloch law below $120 \mathrm{~K}$.

The $T^{3 / 2}$ dependence indicates the presence or existence of spin wave excitations in the ultrafine particles. However, the spin wave constant $B$ was evaluated and is found to be of the order of $10^{-4}$ which is the typical order for nanosized samples as reported by Ping and Lie (1994). For bulk iron this constant is reported to be of the order of $10^{-6}$. Ping and Lie (1994) observed a $B$ value of $10^{-5}$ in $\mathrm{Fe}-\mathrm{SiO}_{2}$ system prepared by sol-gel method. The increase in spin wave constant, $B$, is probably caused by both the finite size effect which causes the softening and cutoff of some spin waves and surface effects that have been confirmed in magnetic thin films (Pierce et al 1982).

The thermomagnetization curve for one representative sample is given in figure 4 . This graph shows a sudden change in magnetization at around $120 \mathrm{~K}$. In order to probe into this particular behaviour, the Mössbauer studies were also carried out on these samples.

\subsection{Mössbauer studies}

The room temperature Mössbauer studies were carried out on some representative samples. These were done both on the cycled and uncyled samples. The low temperature Mössbauer spectra at $150 \mathrm{~K}$ and $100 \mathrm{~K}$ were recorded for the cycled sample.

The ${ }^{57} \mathrm{Fe}$ Mössbauer spectrum of a representative uncycled sample (SRC4) recorded at room temperature is shown in figure 5 .

The Mössbauer spectrum could satisfactorily be fitted with two doublets corresponding to $\mathrm{Fe}^{3+}$ in $\mathrm{A}$ and $\mathrm{B}$ sites. The values of different parameters viz. isomer shift and

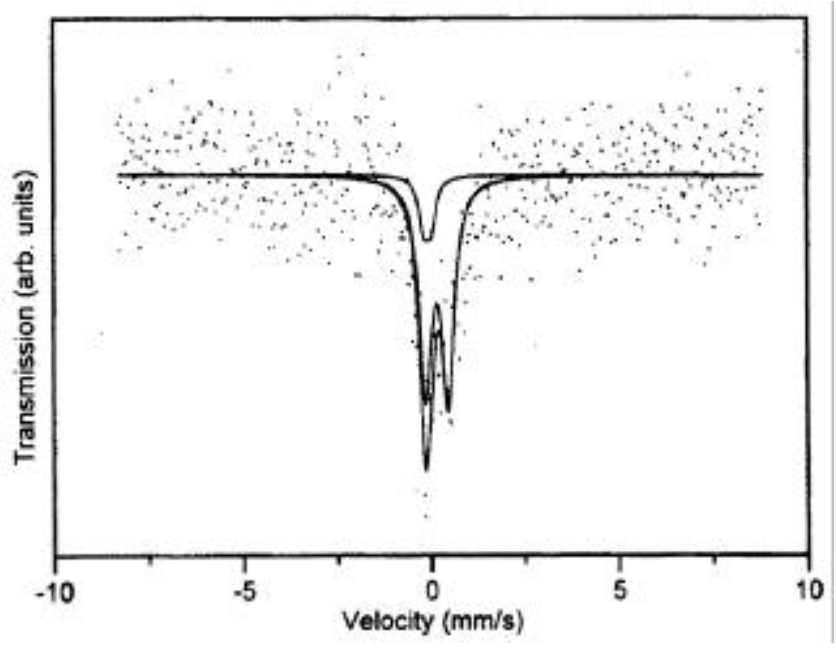

Figure 5. Room temperature Mössbauer spectra of uncycled sample. 
quadrupole splitting, are in agreement with the values reported by Shull et al (1990, 1991), Vassiliou et al (1993), Ziolo et al (1993), Hsu and Huang (1995) and Lukehart et al (1995). The room temperature Mössbauer spectrum for $\gamma-\mathrm{Fe}_{2} \mathrm{O}_{3}$ polymer nanocomposite reported by Shull et al corresponds to a central doublet with an isomer shift of $0.3 \mathrm{~mm} / \mathrm{s}$ and a quadrupole splitting of $0.6 \mathrm{~mm} / \mathrm{s}$.

The Mössbauer spectra of the cycled samples were also recorded at room temperature and low temperatures. The low temperature measurements were carried out at $150 \mathrm{~K}$ and $100 \mathrm{~K}$.

Generally a sample of superparamagnetic material will contain a range of particle size and hence a range of values for $T_{\mathrm{B}}$. So the Mössbauer spectra of the sample will consist of a magnetically split sextet corresponding to large crystallites and a quadrupole split doublet corresponding to smaller crystallites (Chatterjee et al 1990; Shull et al 1990, 1991; Vassiliou et al 1993; Ziolo et al 1993; Hsu and Huang 1995). A clear indication in the Mössbauer for superparamagnetic smaller particles is that the spectral area of the doublet increases at the expense of the sextet with increasing temperature. Also, reports indicate that if the isomer shift is in the range of 0.29$0.33 \mathrm{~mm} / \mathrm{s}$, then they are typical of compounds containing $\mathrm{Fe}^{3+}$ in the octahedral coordination and they are similar to those found in the nanocrystalline sample (Sawatzky et al 1969; Daniels and Rosencwaig 1970; Amulyavichus and Suzdalev 1973; Morup et al 1983, 1995; Chatterjee et al 1990; Gonser et al 1998: Hartridge et al 1998; Lee et al 1998). If there are large quadrupole splitting that is greater than 0.7 they represent very small crystallites. This is because their large surface to volume ratio causes lattice strain and correspondingly large electric field gradient at the iron nuclei. It may also be noted that conventional large crystallites have a quadrupole splitting of $0 \cdot 16$ to $0.8 \mathrm{~mm} / \mathrm{s}$.

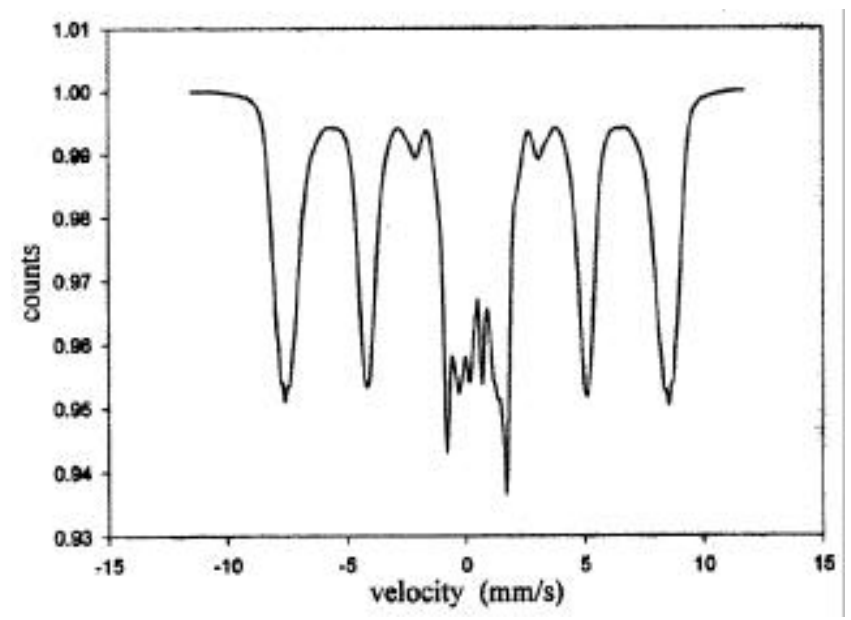

Figure 6. General fit of the room temperature Mössbauer spectra of SA12.
In the present system the room temperature as well as low temperature Mössbauer spectra were recorded for the cycled sample. The general fit of room temperature Mössbauer spectra of SA12 is shown in figure 6 . The spectrum is satisfactorily fitted into a superposition of sharp central doublet and a broad sextet. The fitted doublet and sextet are shown in figures 7 and 8 . The parameters like isomer shift, quadrupole splitting and hyperfine field were obtained from the spectra. They are listed in table 1. From these values it can be seen that the sample consists of a mixture of superparamagnetic and ferromagnetic oxides of iron. The hyperfine field of $501 \mathrm{kOe}$ corresponds to the gamma phase of $\mathrm{Fe}_{2} \mathrm{O}_{3}$. This is in agreement with various reports (Sawatzky et al 1969; Daniels and Rosencwaig 1970; Amulyavichus and Suzdalev 1973; Morup et al 1983, 1995; Chatterjee et al 1990; Gonser et al 1998; Hartridge et al 1998; Lee et al 1998).

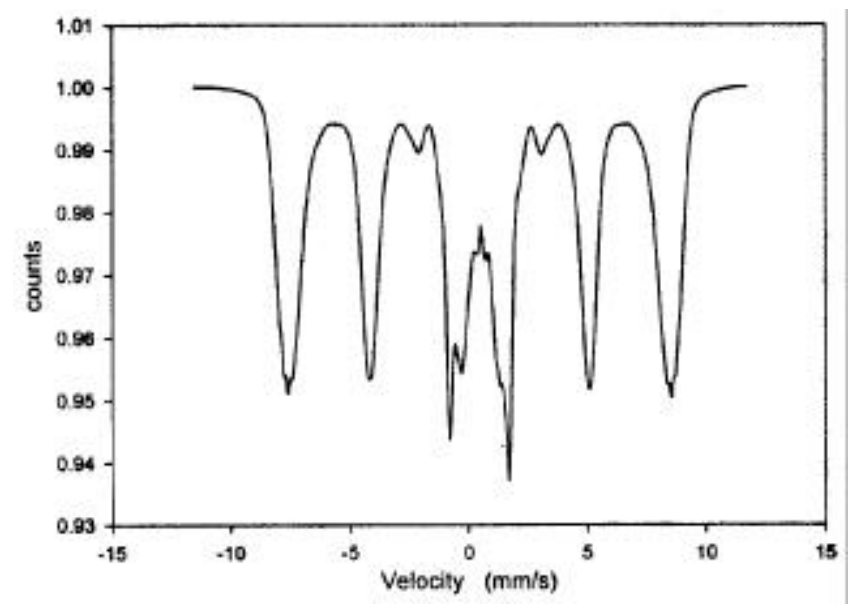

Figure 7. Mössbauer spectrum of the ferrimagnetic component in SA12.

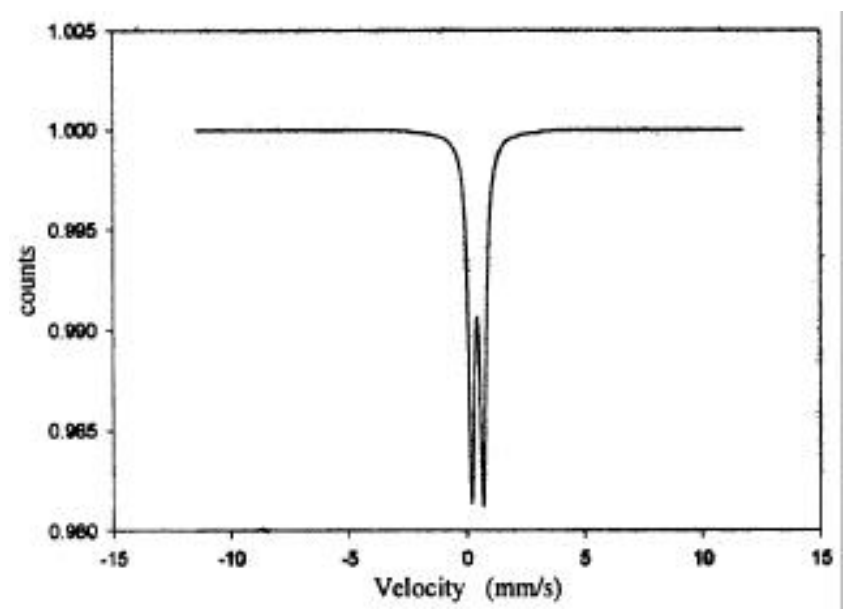

Figure 8. Mössbauer spectrum showing the superparamagnetic component in SA12. 
Table 1. The Mössbauer parameters of the cycled sample.

\begin{tabular}{lcccccc} 
& \multicolumn{3}{c}{ Ferrimagnetic component } & & \multicolumn{2}{c}{ SP component } \\
\cline { 2 - 3 } \cline { 6 - 7 } Temperature & $\begin{array}{c}\text { Isomer } \\
\text { shift } \\
(\mathrm{mm} / \mathrm{s})\end{array}$ & $\begin{array}{c}\text { Quadrupole } \\
\text { splitting } \\
(\mathrm{mm} / \mathrm{s})\end{array}$ & $\begin{array}{c}\text { Hyperfine } \\
\text { field } \\
(\mathrm{kOe})\end{array}$ & & $\begin{array}{c}\text { Isomer } \\
\text { shift } \\
(\mathrm{mm} / \mathrm{s})\end{array}$ & $\begin{array}{c}\text { Quadrupole } \\
\text { splitting } \\
(\mathrm{mm} / \mathrm{s})\end{array}$ \\
\hline $298 \mathrm{~K}$ & 0.47 & 1.2 & $500 \cdot 8$ & & 0.43 & 0.55 \\
$150 \mathrm{~K}$ & 0.47 & 1.2 & $500 \cdot 9$ & & 0.43 & 0.55 \\
$100 \mathrm{~K}$ & 0.47 & 1.2 & $500 \cdot 8$ & & 0.43 & 0.55 \\
\hline
\end{tabular}

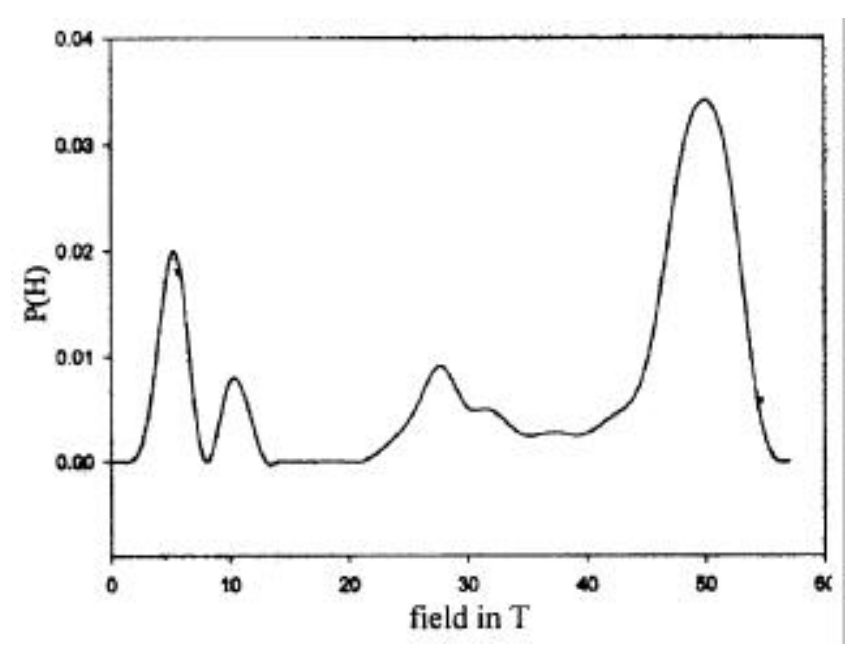

Figure 9. Hyperfine field distribution for SA12 at RT.

The hyperfine field distribution of room temperature spectra is shown in figure 9. From the broad hyperfine field distribution it can be concluded that the magnetic component in the matrix is either in the amorphous form or nanocrystalline form exhibiting superparamagnetic relaxation.

The low temperature Mössbauer spectra were also recorded at $150 \mathrm{~K}$ and $100 \mathrm{~K}$. These spectra were also fitted into superposition of a central doublet and a sextet extending to large velocities. The Mossbauer parameters are shown in table 1.

From table 1 it can be seen that the hyperfine field obtained for the samples at room temperature as well as low temperatures is at around $501 \mathrm{kOe}$ which is again characteristic of gamma iron oxide. The isomer shift and quadrupole splitting for the SP component at all temperatures is around $0.43 \mathrm{~mm} / \mathrm{s}$ and $0.55 \mathrm{~mm} / \mathrm{s}$, respectively which are characteristic of nanosized iron oxide (Shull et al 1990, 1991; Vassiliou et al 1993; Ziolo et al 1993; Hsu et al 1995). Thus from the room temperature and low temperature Mössbauer spectra it can be seen that the magnetic component may be in the nanocrystalline form exhibiting superparamagnetic relaxation. At low temperatures of $150 \mathrm{~K}$ and $100 \mathrm{~K}$ the hyperfine field considerably narrows down with only one peak around $50 \mathrm{~T}$. This also suggests that probably the broad hyperfine field distribution at room temperature is because of superparamagnetic relaxation of nanoparticles.

The mechanical coupling of small particles to its surrounding can also be studied by using Mössbauer since the thermal vibrations cause a lowering of the recoil free fraction (Morup et al 1995). Temperature dependence of relative area of the Mössbauer spectra will give an idea about the same which will be undertaken later as a continuation of this work.

\section{Conclusions}

Low temperature magnetization and Mössbauer effect studies carried out on selected nanocomposite samples indicate that the composites consist of very fine particles of $\gamma-\mathrm{Fe}_{2} \mathrm{O}_{3}$. A definite amount of this iron oxide exists in the superparamagnetic phase. The evaluation of the Bloch constant and its numerical value suggests that the particles are definitely in the superparamagnetic state. However, deviations from Bloch equation at temperatures below $120 \mathrm{~K}$ points to surface effects caused by finite sizes of the particle which causes the softening and cut off of the spin waves and this warrants further investigations. Mössbauer spectroscopic studies give conclusive evidence for the existence of $\gamma-\mathrm{Fe}_{2} \mathrm{O}_{3}$ phase in the nanocomposites. Cycling increases the amount of ferromagnetic component in the nanocomposites. The broad hyperfine field distribution noticed in the Mössbauer spectrum is indicative of superparamagnetic relaxation.

\section{Acknowledgements}

Authors thank IUCDAEF, Indore, for recording the Mössbauer spectra. (MRA) thanks DST, New Delhi, for financial assistance received in the form of a project. (KAM) thanks the Council of Scientific and Industrial Research, New Delhi, for a Research Fellowship.

\section{References}

Ambrose T, Gavrin A and Chien C L 1992 J. Mag. Mag. Mater. 116 L 311 
Amulyavichus A P and Suzdalev I P 1973 Sov. Phys. JETP 37 859

Bean C P 1955 J. Appl. Phys. 261381

Bean C P and Livingstone J D 1959 J. Appl. Phys. 30120

Chakravorty D 1992 Bull. Mater. Sci. 15411

Chatterjee A, Das D, Chakravorty D and Choudhury K 1990 Appl. Phys. Lett. 571360

Dagani R 1992 Nanostructured materials promise to advance range of technologies, scienceltechnology (American Chemical Society: Chemical and Engineering News) pp 18-24

Daniels J M and Rosencwaig A 1970 Canadian J. Phys. 48380

Gonser U, Weidersich H and Grant R W 1998 J. Appl. Phys. 39 1004

Hartridge A, Bhattacharya A K, Majumdar C K, Das D and Chintalapudi S N 1998 J. Mag. Mag. Mater. 183 L1

Hsu Jen-Hwa and Huang Yi-Hong 1995 J. Mag. Mag. Mater. 140-144 405

Lee J G, Park J Y and Kim C S 1998 J. Mater. Sci. 333965

Lukehart C M, Milne S B, Stock S R, Shull R D and Witting J E 1995 Mater. Sci. Engg. A204 176

Malini K A, Anantharaman M R, Sindhu S, Chinnasamy C N, Ponpandian N, Narayanasamy A, Balachandran M and Sivasankara Pillai V N 2001 J. Mater. Sci. 36821

Morup S 1994 Europhys. Letts 28671
Morup S, Madsen M B, Franck J, Villadsen J and Koch C J W 1983 J. Mag. Mag. Mater. 40163

Morup S, Oxborrow C A, Hendriksen P V, Pedersen M S, Hanson M and Johansson C 1995 J. Mag. Mag. Mater. 140-144 409

Ping W J and Lie L H 1994 J. Mag. Mag. Mater. 13154

Pierce D T, Celotta R J, Unguris J and Seigmann H C 1982 Phys. Rev. B26 2566

Roy S, Das D and Chakravorty D 1993 J. Appl. Phys. 74474

Sawatzky G A, Vander Woude F and Morrish A H 1969 Phys. Rev. 187747

Shull R D and Bennet L H 1992 J. Nanostruct. Mater. 183

Shull R D, Ritter J J, Shapiro A J, Swartzendruder L J and Bennet L H 1990 J. Appl. Phys. 674490

Shull R D, Ritter J J, Shapiro A J, Swartzendruder L J and Bennet L H 1991 MRS symposium proc. (Materials Research Society) 206455

Vassiliou J K, Mehrotra V, Russell M W, Giannelis E P, Mcmicheal R D, Shull R D and Ziolo R F 1993 J. Appl. Phys. 735109

Ziolo R F, Giannelis E P, Weinstein B A, Michela P, O'Horo, Ganguly B N, Mehrotra V, Russel M W and Huffman D R 1992 Science 257219

Ziolo R F, Giannelis E P and Shull R D 1993 Nanostruct. Mater. 395 